\title{
Mitochondrial DNA rearrangements in young onset parkinsonism: two case reports
}

\author{
G Siciliano, M Mancuso, R Ceravolo, V Lombardi, A Iudice, U Bonuccelli
}

\begin{abstract}
Parkinson's disease is a nosological entity of unknown origin for which, in some cases, a possible pathogenetic role for mitochondrial dysfunction has been postulated. Two young onset parkinsonian patients with mitochondrial DNA (mtDNA) deletions in skeletal muscle are reported on. Patient 1 also presented with increased blood creatine kinase and lactate concentrations and a family history which included a wide range of phenotypes affecting multiple systems. Patient 2 presented with multiple symmetric lipomatosis. Histopathological investigation showed ragged red fibres and COX negative fibres in muscle biopsies from both patients. The data support the hypothesis that mitochondrial DNA mutations may occur in some cases of parkinsonism, suggesting that a diagnosis of a mitochondrial disorder should be considered in the presence of consistent family history and clinical symptoms. (f Neurol Neurosurg Psychiatry 2001;71:685-687)
\end{abstract}

Keywords: Parkinson's disease; mitochondrial encephalomyopathies; mtDNA deletions

Mitochondrial diseases comprise a wide group of disorders which, at their origin, have nuclear or mitochondrial DNA (mtDNA) mutations, which are responsible for biochemical defects in respiratory chain function and consequent impaired cellular oxidative energy production. It follows that mitochondrial disorders are associated with a wide group of clinical manifestations affecting skeletal muscle, brain, heart, and other highly oxygen dependent organs or tissues. ${ }^{1}$

The hypothesis of pathogenic mitochondrial dysfunction in idiopathic Parkinson's disease has become particularly attractive since the finding by Vyas et al that the parkinsonism inducing compound N-methyl-4-phenyl1,2,3,6-tetrahydropyridine is a mitochondrial toxin. ${ }^{2}$ After further reports on deficiency in respiratory chain enzyme complex I in the substantia nigra of patients with Parkinson's disease, ${ }^{3}$ other investigators have tried to clarify the relation between mitochondrial defects and death of nigral dopaminergic neurons. ${ }^{4}$
Although the precise role of respiratory chain dysfunction and mtDNA mutations in the pathogenesis of Parkinson's disease remains to be elucidated, it is worth noting that in most series of patients with a primary defined defect in mitochondrial activity, akinetic rigid syndromes are infrequent. ${ }^{5}$

Conversely, recent reports indicate the occurrence of primary pathogenic mtDNA mutations in Parkinson's disease, both in the form of large scale mtDNA rearrangements, ${ }^{6}$ and as point mutations in complex $\mathrm{I}^{7}$ and tRNA genes. $^{8}$

We report here on two unrelated patients in whom parkinsonism was part of peculiar clinical constellations. In these patients molecular analysis showed the presence of major mtDNA rearrangements in skeletal muscle, supporting the hypothesis that the dopaminergic nigrostriatal system can be a target, at least in some instances, of mitochondrial involvement.

\section{Case reports}

CASE 1

A 40 year old man was referred to us because of young onset (at 30 years) left lateral parkinsonism responsive to levodopa. Neurological examination showed bradykinesia and rigidity, postural instability, and hypomimia (Hoehn and Yahr stage III). Tremor, pyramidal signs, dysautonomia, oculomotor dysfunction, cerebellar impairment, and orthostatic hypotension were absent. Therapy, at that time, was $600 \mathrm{mg} /$ day levodopa/carbidopa and $300 \mathrm{mg} /$ day tolcapone. Motor fluctuations in the form of wearing off were present. General physical examination was normal, and no Kayser-Fleischer rings were present on ophthalmological examination. Routine laboratory blood chemistry showed abnormal concentrations of creatine kinase (901 U/1; normal value<195), glutamic oxaloacetic transaminase $(76 \mathrm{U} / 1$; normal value $<45)$, glutamic pyruvic transaminase $(170 \mathrm{U} / 1$; normal value $<45)$, and $\gamma$-glutamyltransferase $(73 \mathrm{U} / 1$; normal value $<50 \mathrm{U} / \mathrm{l}$ ), whereas blood ceruloplasmin and urinary copper concentrations were normal.

Abdominal ultrasound scan showed liver cirrhosis and tolcapone was subsequently withdrawn. Blood lactate concentration was abnormally increased during progressive workload exercise (increment by $120 \%$ of the basal value at $50 \%$ of maximal power output'; normal 


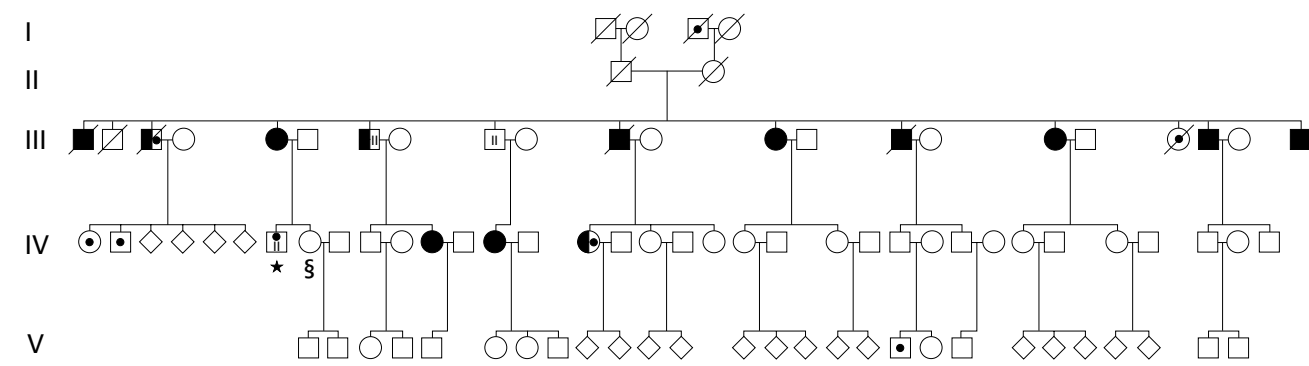

Figure 1 Pedigree of patient 1, square=male; circle=female; $/=d e a d$; diamond=unknown sex. Empty symbols=non-affected members; black symbols=affected by diabetes mellitus; point symbols=affected by liver cirrhosis; II=affected by Parkinson's disease; $\int=$ proband; $*=$ case 1 .

value $<50 \%$ ). Brain MRI was normal, as was neuropsychological evaluation. Liver cirrhosis, diabetes mellitus, and a complex pattern of neurological disorders were disclosed on examination of family members (fig 1). In particular, the patient's 48 year old sister, the proband of this family, had been affected by bilateral eyelid ptosis, progressive external ophthalmoplegia, and sensory ataxia since her early 40 s, and increased concentrations of creatine kinase $(406 \mathrm{U} / \mathrm{l})$ and rest lactate $(56.7 \mathrm{mg} / \mathrm{dl}$, normal value 5.7-22). Furthermore, family medical history disclosed a history of parkinsonism in two maternal uncles, and other maternally related members were affected by liver cirrhosis and diabetes mellitus, a clinical association thought to be responsible for the death of three of the patient's relatives in their 50s.

Due to the family history a left deltoid muscle biopsy under local anaesthesia was performed on the patient, after obtaining informed consent. Increased variability in fibre size, scattered $(3 \%)$ ragged red fibres by trichrome and $10 \%$ COX negative fibres were found on histochemical examination. Whereas polymerase chain reaction (PCR) analysis of mtDNA was negative for A8344G and G8363A MERRF and for A3243G and A3252G MELAS point mutations, Southern blot disclosed, in addition to a wild-type band of $16.6 \mathrm{~kb}$, at least four additional bands corresponding to multiple species of deleted mtDNA. The densitometric amount of mutant mtDNAs was about $60 \%$ of total mtDNA. About $8 \%$ of ragged red and COX negative fibres and a similar pattern of mtDNA rearrangement were found in a muscle biopsy from the patient's sister.

\section{CASE 2}

A 52 year old man came to our attention with a history of young onset (38 years) parkinsonism responsive to levodopa. On physical examination he showed multiple, symmetric fatty proliferations on his neck, shoulders, and limbs, with subcutaneous lipomas visible on ultrasound scan. Neurological examination showed upper limb rest tremor, bradykinesia, rigidity lateralised on the left side, and postural instability (Hoehn and Yahr stage III). The remaining neurological examination was unremarkable. The patient's treatment at the time of referral was $400 \mathrm{mg} /$ day levodopa/ benserazide and $6 \mathrm{mg} /$ day ropirinole. Motor fluctuations of the wearing off type were present. Routine laboratory blood chemistry was normal. Blood lactate concentration at rest was slightly increased above the normal range (25 mg/dl; normal value 5.7-22), and brain MRI was normal. Family history was negative for neurological disorders, including symptoms and signs more commonly suggestive of mitochondriopathy. Muscle biopsy showed increased variability in fibre size and scattered ragged red and COX negative fibres. Polymerase chain reaction analysis of mtDNA was negative for the above mentioned MERRF and MELAS point mutations. Long PCR showed, in addition to wild-type mtDNA, one additional band, of about $11.6 \mathrm{~kb}$, corresponding to a 5000 bp mtDNA deletion (fig 2) located, according to the primers used, between nucleotides 8420 and 13648 (common deletion). The amount of mutant mtDNA was about $50 \%$ of total mtDNA.

\section{Discussion}

In this study we have described two patients with a history of parkinsonism and the presence of mitochondrial DNA (mtDNA) mutations in skeletal muscle. These two patients showed some peculiarities in their clinical picture - namely, a complex and variable occurrence of symptoms suggestive of diseases caused by mtDNA mutations. In particular we recorded progressive external ophthalmoplegia and diabetes mellitus in the family of patient 1 , and in patient 2 , multiple symmetric lipomatosis, a disorder often sustained by mitochondrial impairment. ${ }^{10}$ Moreover, in family 1 the clinical findings seemed to be inherited in autosomal dominant fashion and they were associated with multiple mtDNA deletions in the two analysed members belonging to a same generation, making it possible for an autosomal dominant mutation to cause such an mtDNA abnormality. ${ }^{11}$

Causative factors of selective degeneration of nigrostriatal neurons in Parkinson's disease remain largely unknown. However, there is increasing evidence in favour of a significant genetic component in the pathogenesis of this disease, as recently indicated by the finding of a point mutation in the $\alpha$-synuclein gene in some pedigrees with autosomal dominant Parkinson's disease, ${ }^{12}$ or parkin gene deletions in some patients with autosomal recessive Parkinson's disease. ${ }^{13}$

Among the possible genetic determinants of Parkinson's disease, the mitochondrial genome has also been considered. Although parkinsonism is not a common part of the constellation 
of symptoms that accompanies mitochondrial syndromes, ${ }^{5}$ a heteroplasmic G5460A mutation affecting the ND2 subunit of $\mathrm{NADH}$ dehydrogenase has been detected in several brains of patients with idiopathic Parkinson's disease $^{14}$ and novel point mutations of mitochondrial complex $\mathrm{I}^{7}$ and tRNA(Thr) and tRNA(Pro) ${ }^{8}$ genes have been identified in pathologically confirmed Parkinson's disease. The possibility that as yet unidentified polymorphisms within the mitochondrial genome are relevant to the complex I defect found cannot be excluded, and a recent paper ${ }^{15}$ has provided evidence that this may be the case. Some studies have also investigated the possible relation between Parkinson's disease and the presence of the 4977 base pair mtDNA "common deletion" in tissues from patients, concluding that the pathogenic role of large scale mtDNA deletions in Parkinson's disease is still controversial. ${ }^{16}$ Therefore, current opinion is that mitochondrial impairment could be one of the factors, but not necessarily the primary or the major one, which intervene in the sequence of events involved in the pathogenesis of Parkinson's disease.

In both the patients reported here the reasons for selective involvement of the nigrostriatal system as a main manifestation of mitochondrial disease are speculative. According to general principles of mitochondrial genetics this could be interpreted as a consequence of random tissue segregation of mutated $\mathrm{mtDNA}$, but also as the effect of the high vulnerability of those tissues that are mainly dependent on aerobic metabolism for their function. ${ }^{1}$

In conclusion, this study suggests that a diagnosis of a mitochondrial disorder should be considered in those parkinsonian patients in whom the concurrence of consistent family history and clinical constellation of symptoms occurs.
The financial support of Telethon-I (project No 1179 c) is gratefully acknowledged.

1 Di Mauro S, Schon EA. Mitochondrial DNA and diseases of the nervous system. The spectrum. Neuroscientist 1997; 4:53-63.

2 Vyas I, Heikkila RE, Nicklas WJ. Studies on the neurotoxicity of 1-methyl-4-phenyl-1,2,5,6-tetrahydropyridine: inhibition of NAD-linked substrate oxidation by its metabolite, 1-methyl-4-pyridinium. F Neurochem 1986;46:1501-7.

3 Schapira AHV, Cooper JM, Dexter D, et al. Mitochondrial complex I deficiency in Parkinson's disease. Lancet 1989; 1269.

4 Mizumo Y, Ikebe S, Hattori N, et al. Role of mitochondria in etiology and pathogenesis of Parkinson's disease. Biochim Biophys Acta 1995;1271:265-74.

5 Truong DD, Harding AE, Scaravilli F, et al. Movement disorders in mitochondrial myopathies. A study of nine cases with two autopsy studies. Mov Disord 1990;5:109-17.

6 Chalmers RM, Brockington M, Howard RS, et al. Mitochondrial encephalopathy with multiple mitochondrial DNA deletions: a report of two families and two sporadic cases with unusual clinical and neuropathological features. $\mathcal{7}$ Neurol Sci 1996;143:41-5.

7 Kosel S, Grasbon-Frodl EM, Mautsch U, et al. Novel mutations of mitochondrial complex I in pathologically proven tions of mitochondrial complex I in pathological

8 Grasbon-Frodl EM, Kosel S, Sprinzl M, et al. Two novel point mutations of mitochondrial tRNA genes in histologically confirmed Parkinson disease. Neurogenetics 1999;2: $121-7$.

9 Siciliano G, Renna M, Manca ML, et al. The relationship of plasma catecholamine and lactate during anaerobic threshold exercise in mitochondrial myopathies. Neuromuscul Disord 1999;9:411-16.

10 Klopstock T, Naumann M, Seibel P, et al. Mitochondrial DNA mutations in multiple symmetric lipomatosis. Mol Cell Biochem 1997;174:271-5.

11 Zeviani M, Servidei S, Gellera C, et al. An autosomal dominant disorder with multiple deletions of mitochondrial DNA starting at the D-loop region. Nature 1989;339:30911

12 Polymeropoulos MH, Lavedan C, Leroy E, et al. Mutation in the alpha-synuclein gene identified in families with Parkinson's disease. Science 1997;276:2045-7.

13 Lucking CB, Abbas N, Durr A, et al. Homozygous deletions in parkin gene in European and North African families with autosomal recessive juvenile parkinsonism. Lancet with autosomal rece

14 Schnopp NM, Kosel S, Egensperger R, et al. Regional heterogeneity of mtDNA heteroplasmy in parkinsonian brain. Clin Neuropathol 1996;15:348-52.

15 Swerdlow RH, Parks JK, Miller SW, et al. Origin and functional consequences of the complex I defect in Parkinson's disease. Ann Neurol 1996;40:663-71.

16 Kosel S, Egensperger R, Schnopp NM, et al. The common deletion is not increased in parkinsonian substantia nigra as shown by competitive polymerase chain reaction Mov Disord 1997;12:639-45. 